This item was submitted to Loughborough's Research Repository by the author.

Items in Figshare are protected by copyright, with all rights reserved, unless otherwise indicated.

A comparison of modelling approaches for the time-limited dispatch (TLD) of aircraft

PLEASE CITE THE PUBLISHED VERSION

PUBLISHER

(C) IMechE / Professional Engineering Publishing

LICENCE

CC BY-NC-ND 4.0

REPOSITORY RECORD

Prescott, Darren R., and J.D. Andrews. 2008. "A Comparison of Modelling Approaches for the Time-limited Dispatch (TLD) of Aircraft". figshare. https://hdl.handle.net/2134/3942. 
This item was submitted to Loughborough's Institutional Repository (https://dspace.lboro.ac.uk/) by the author and is made available under the following Creative Commons Licence conditions.

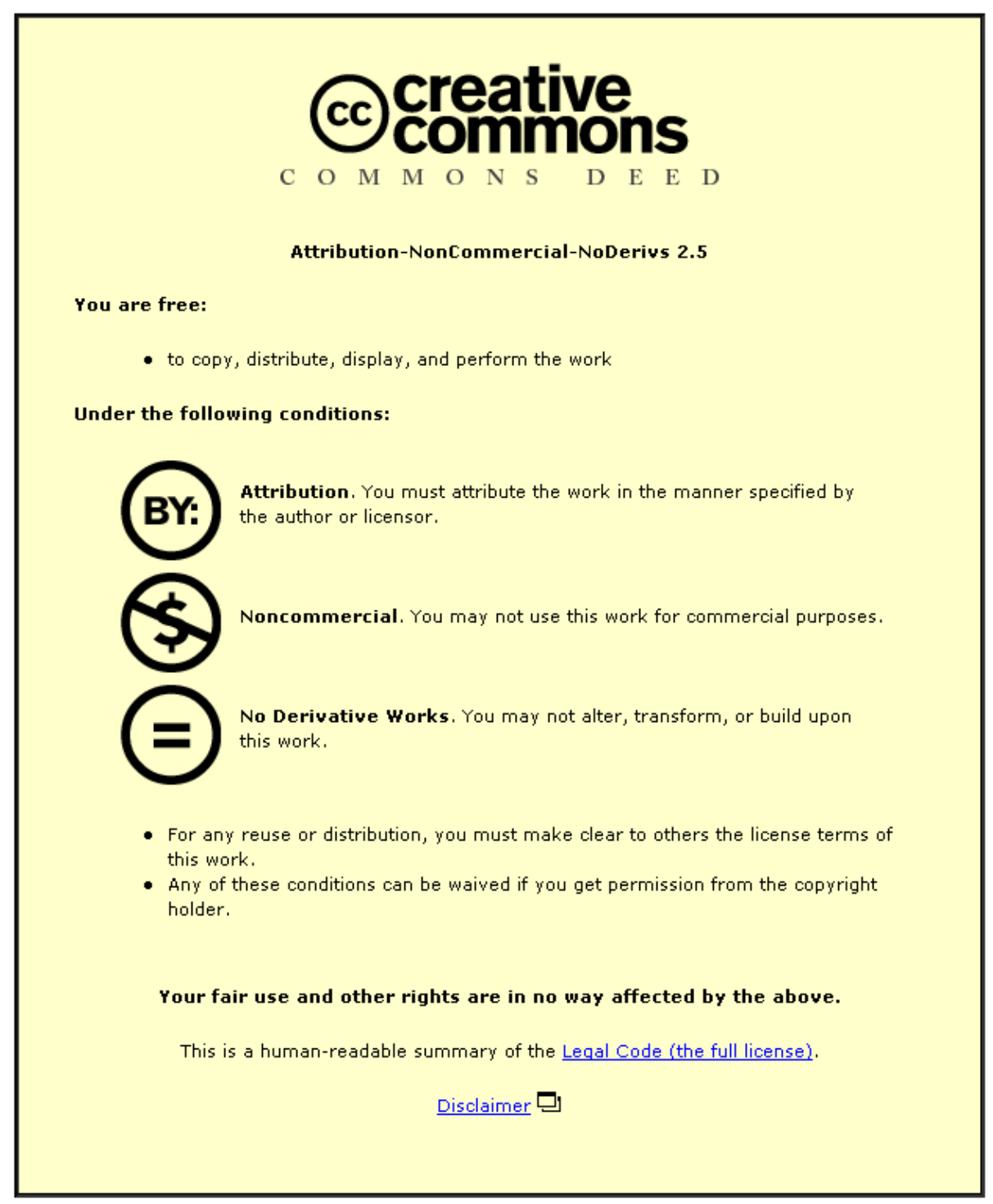

For the full text of this licence, please go to: http://creativecommons.org/licenses/by-nc-nd/2.5/ 


\title{
A comparison of modelling approaches for the time-limited dispatch (TLD) of aircraft
}

\author{
D Prescott and J D Andrews* \\ Department of Aeronautical and Automotive Engineering, Loughborough University, Loughborough, UK
}

The manuscript was received on 15 June 2005 and was accepted after revision for publication on 8 March 2006.

DOI: 10.1243/1748006XJRR9

\begin{abstract}
The time-limited dispatch (TLD) of aircraft allows operators efficiently to meet certification requirements. In order to show that these requirements are met it is necessary to model the aircraft systems to which TLD is being applied. Currently, variations of fault tree analysis and Markov analysis are commonly used. However, in order to apply either of these methods, a number of assumptions are made to assist in the analysis. Monte Carlo simulation (MCS) is presented here as an alternative method of demonstrating the required level of system reliability. A simple system is analysed using a time-weighted average approach, a reduced fault state Markov approach, and an MCS approach. MCS is seen to offer benefits when modelling the application of TLD to a simple system that could also be seen in the modelling of the application of TLD to real aircraft systems.
\end{abstract}

Keywords: time-limited dispatch, Monte Carlo simulation

\section{INTRODUCTION}

Time-limited dispatch (TLD) was first utilized after the introduction of full authority digital electronic control (FADEC) systems to commercial aircraft some 20 years ago. These electronic engine control systems regulate engine thrust from the beginning of fuel metering up to the time of fuel shut-off. When FADEC systems were introduced, it was to be the first time that a hydromechanical control (HMC) system would be unavailable to pilots in the event of an electronic system failure [1].

FADEC systems are designed around a dual channel control system, and, as such, incorporate a degree of redundancy. Each engine has a FADEC system in which all critical loops and functions have either dual systems or redundant elements. Although it was expected that this would lead to greater control system integrity, the dispatch criteria imposed when FADECs were introduced actually led to an increase in delays and cancellations of flights [2], [3]. This was due to the fact that, in the absence

*Corresponding author: Department of Aeronautical and Automotive Engineering, University of Loughborough, Loughborough, Leicestershire LE11 3TU, UK. email: J.D.Andrews@ lboro.ac.uk of any dispatch guidelines for FADEC systems, a conservative approach was taken in which dispatch was forbidden with faults in more than one channel of an engine. However, because of the high reliability of the FADEC systems in comparison to the HMC systems, an opportunity arose to utilize the redundancy present to allow dispatch with faults present in the FADECs. Required airworthiness standards would still be met and aircraft operators would benefit from the reduction in delays and cancellations of flights. The new approach, which allowed dispatch with reduced levels of redundancy, was called timelimited dispatch (TLD).

TLD allows the dispatch of aircraft with faults present while ensuring a level of system reliability. This level was set according to the levels that were required of the HMC systems that were used prior to the introduction of FADECs. A maximum limit of 10 events per $10^{6}$ flight hours (flt $\mathrm{h}$ ) is set for the average loss of thrust control (LOTC) rate of the system [2]. In achieving this average, a further restriction of an upper limit of 100 events per $10^{6} \mathrm{flt} \mathrm{h}$ is applied for the instantaneous LOTC rate of dispatchable system configurations.

When implementing TLD an aircraft may be dispatched over differing periods of time according to the significance of the faults present in the system 
[2]. These dispatch intervals, which give the maximum time allowed for dispatch before the faults must be addressed, fall into four categories:

(a) do not dispatch (DND);

(b) short time dispatch (STD);

(c) long time dispatch (LTD);

(d) manufacturer/operator defined dispatch (MDD).

The DND category, when applied, means the faults present in the system prohibit dispatch of the aircraft and the faults must be addressed immediately. The STD category allows operation of the aircraft in the short term before corrective maintenance must be undertaken and the LTD category allows dispatch in the longer term. The final category, MDD, is reserved for faults that do not affect the LOTC rate of the system [2]. The LOTC rate for faults in the LTD category must not exceed 75 events per $10^{6} \mathrm{flt}$ h. STD category faults have an LOTC rate that lies between 75 and 100 events per $10^{6} \mathrm{flt} \mathrm{h}$ and for DND category faults the instantaneous LOTC rate exceeds 100 events per $10^{6} \mathrm{flt} \mathrm{h}$.

\subsection{Maintenance strategies}

Two maintenance strategies exist that may be used to maintain a system on which TLD is being implemented. There is no restriction to which strategy must be used when maintaining a system. In fact, if desired, one of the strategies may be used to maintain STD category faults while the other is used to maintain LTD category faults. The two maintenance approaches are described below.

\subsubsection{MEL maintenance}

Minimum equipment list (MEL) maintenance [2] is generally applied to STD faults. When MEL maintenance is used, the exact time of occurrence of the fault must be known, at which time a 'countdown' is started of the appropriate dispatch interval. When the countdown ends, the fault must be repaired in order to allow further dispatch of the aircraft. This process is illustrated in Fig. 1, where a fault occurring at time $t_{1}$ initiates a dispatch interval ending at $t_{2}$. If the fault is not repaired at or before $t_{2}$, further dispatch of the aircraft is prohibited at that time.

\subsubsection{PIR maintenance}

The second method of maintenance is periodic inspection/repair (PIR) maintenance, normally used with LTD category faults. This involves checking the system for faults at regular intervals. In this case the exact time of occurrence of the fault will not be known. If a fault is discovered at an

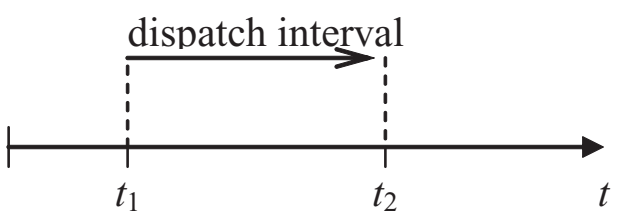

Fig. 1 MEL maintenance

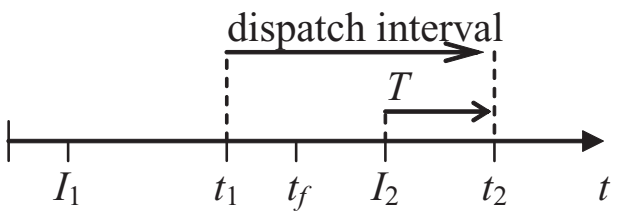

Fig. 2 PIR maintenance

inspection, it is assumed to have occurred at the midpoint of consecutive inspections [2]. The dispatch interval is then deemed to have begun at the midpoint of the inspections and the allowed period of dispatch from the inspection where the fault was discovered is calculated. A PIR maintenance scenario is illustrated in Fig. 2, in which $I_{1}$ and $I_{2}$ represent consecutive inspections of the system for faults. A fault, which occurred at time $t_{\mathrm{f}}$ is discovered at $I_{2}$ and, because the exact time of the fault is not known, is assumed to occur at $t_{1}$, the midpoint of the inspection interval. The countdown of the dispatch interval is then assumed to have begun at this time and it will end at $t_{2}$. This allows dispatch of the aircraft for a further time $T$ after $I_{2}$. The inspection interval for a fault category must not exceed twice the dispatch interval for faults of that category. In that way the average exposure to faults cannot exceed the appropriate dispatch interval. However, note that the maximum possible exposure of the system to a fault could be twice the dispatch interval. Contrast this with the MEL maintenance approach where the maximum possible exposure of the system to a fault is equal to the dispatch interval.

PIR could be used to address the maintenance requirements of more than one category of faults. In systems where this is the case, inspections for faults of a certain category may uncover faults that fall into another dispatch category. If this occurs, the fault may be dealt with as if discovered at the next inspection for its own category [2]. For example, the presence of an STD fault at an LTD inspection would be noted but the STD fault would be treated as if found at the next inspection for STD faults.

\subsection{The simultaneous presence of multiple faults in the system}

In spite of the high reliability of FADEC system components, there exists the opportunity for more than 
one fault to be present in the system at any one time. If this happens, there are a number of different issues that could arise and impact upon the maintenance of the FADEC system. Examples of such scenarios are outlined below for the MEL maintenance strategy. These examples are by no means exhaustive, but merely hint at the complexities involved in modelling the TLD process. Indeed, when one begins to consider PIR maintenance or a combination of MEL and PIR and, although such situations would be rare, the presence of more than two faults, the maintenance options available become more complex.

Consider Fig. 3, which depicts the occurrence of two faults, $A$ and $B$, repaired using MEL maintenance. The dispatch intervals for these faults will end at $t_{1}$ and $t_{2}$ respectively. As $t_{1}$ is reached, a number of options are possible. Clearly, fault $A$ must be cleared from the system at this time in order to allow further dispatch. In addition to this either:

(a) $B$ may be allowed to remain in the system, allowing dispatch until $t_{2}$; or

(b) $B$ may also be cleared from the system, allowing unlimited dispatch after $t_{1}$.

Figure 4 depicts a similar scenario to that shown in Fig. 3. Faults $A$ and $B$, when occurring in isolation, cause the initiation of LTD intervals, ending at $t_{1}$ and $t_{2}$ respectively. However, as soon as both $A$ and $B$ are present within the system, the allowable period of dispatch is reduced to the STD category. This means that, as fault $B$ occurs, the system may then be dispatched only until time $t_{3}$, not $t_{2}$ as would be the case if fault $A$ had not occurred. Upon reaching $t_{3}$, there are three possible maintenance strategies.

1. Both faults, $A$ and $B$, may be cleared from the system, allowing unlimited dispatch of the system.

2. Fault $B$ alone may be cleared from the system, allowing dispatch until $t_{1}$, at which point fault $A$ must be addressed.

3. Fault $A$ alone may be cleared from the system, allowing dispatch until $t_{2}$, at which point fault $B$ must be addressed.

Of course, this scenario assumes that $t_{3}$ occurs before $t_{1}$. If fault $B$ occurred at such a time that $t_{3}$ occurred after $t_{1}$, then fault $A$ would have to be cleared from the system at $t_{1}$ before the STD maintenance deadline was reached at $t_{3}$.

When faults combine in the way just described to reduce the dispatch interval, the order of occurrence of the faults may have an effect on whether or not the dispatch interval is reduced. For instance, in the example shown in Fig. 4 , if fault $B$ follows fault $A$, an STD interval is initiated. If the ordering of these faults was unimportant, the same reduction

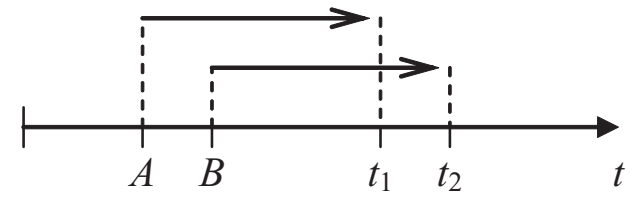

Fig. 3 Multiple faults (MEL maintenance)

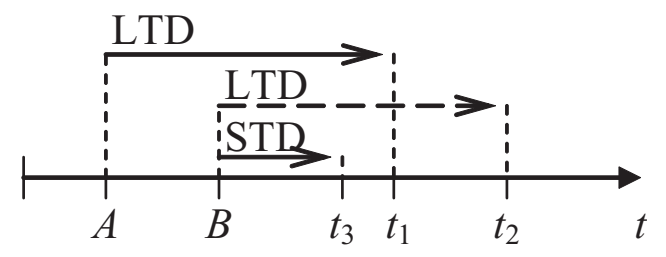

Fig. 4 The combination of multiple faults (MEL maintenance)

in dispatch interval would be seen if fault $A$ followed fault $B$. However, if the ordering of these faults was important, it may be that $A$ following $B$ would not lead to the same reduction in dispatch interval as when $B$ followed $A$.

The scenarios described above merely hint at the complexities of TLD and the repair processes involved in the maintenance of a FADEC system. When analysing the use of TLD in the maintenance of a FADEC system, the ability of the model used to deal with these complexities may be of importance if accurate results are to be obtained.

\section{MODELLING TLD}

Before applying TLD to a FADEC system, it is important to be sure that the system will still meet the levels of safety required of it. To do this a mathematical model of the system is constructed and an analysis is performed to monitor the effects of TLD on the system and obtain the average LOTC rate of the system. Two methods of analysis that are widely used are described in references [1] and [3]. These approaches are based on fault tree analysis (FTA) and Markov analysis and are described briefly below. Also described is a third technique, Monte Carlo simulation (MCS), proposed by the authors as a suitable alternative method of conducting a TLD analysis.

\subsection{Time-weighted average (TWA) approach}

This TLD modelling approach obtains a value for the LOTC rate of the system by adding the following three quantities:

(a) the sum of the failure rates of faults in the mechanical/hydromechanical portion of the FADEC system; 
(b) the sum of the failure rates of the system owing to unrevealed electrical/electronic faults;

(c) a time-weighted average (TWA) of the failure rates of the system from each of its dispatchable configurations.

This last quantity is obtained by multiplying the fraction of time spent in each dispatchable system configuration by the failure rate to LOTC from that particular configuration. Consider a FADEC system with $n$ dispatchable configurations and let the first of these (configuration 1) represent the full-up system state; the configurations numbered from 2 to $m$ represent the configurations allowing STD and the configurations from $m+1$ to $n$ represent the configurations allowing LTD. Let $\lambda_{i}$ represent the failure rate into the $i$ th dispatchable configuration. Define $T_{i}$, the dispatch interval for the $i$ th dispatchable system configuration as follows

$$
T_{i}= \begin{cases}T_{\mathrm{STD}}, & \text { if } i=2, \ldots, m \\ T_{\mathrm{LTD}}, & \text { if } i=m+1, \ldots, n\end{cases}
$$

where $T_{\mathrm{STD}}$ is the short time dispatch interval and $T_{\text {LTD }}$ is the long time dispatch interval. Thus an expression for the TWA LOTC rate of the system is

$$
\lambda_{\mathrm{TWA}}=\lambda_{\mathrm{HMC}}+\lambda_{\mathrm{UR}}+\sum_{i=1}^{n} t_{i} \lambda_{i, L}
$$

where $\lambda_{\text {HMC }}$ represents the sum of failure rates due to mechanical/hydromechanical faults and $\lambda_{\mathrm{UR}}$ represents the sum of failure rates due to unrevealed electrical/electronic faults. The fraction of time spent dispatching from dispatchable configuration $i$ is $t_{i}$ and $\lambda_{i, \mathrm{~L}}$ represents the system failure rate to LOTC from the $i$ th dispatchable configuration. Equation (2) is a general form of the equations given in references [1], [3], and [4]. Because $t_{i}$ represents the fraction of time spent dispatching from system state $i$, it is required that the total of all $n$ of these fractions is unity. Thus, the following expression for $t_{1}$, the fraction of time spent dispatching from the full-up state, is obtained

$$
t_{1}=1-\sum_{i=2}^{n} t_{i}
$$

An approximation for $t_{i}$ with $i=2, \ldots, n$, i.e. the fraction of time spent dispatching from the faulty dispatchable configurations, is

$$
t_{i}=\lambda_{i} T_{i}
$$

This is equivalent to the approximation given in the original version of SAE ARP5107 [3].

The system failure rate to LOTC from the $i$ th dispatchable system configuration, $\lambda_{i, \mathrm{~L}}$, is calculated in reference [1] by dividing the failure probability to LOTC by the average flight time. This 'probability per flight hour' is equated to the desired failure rate. Thus

$$
\lambda_{i, \mathrm{~L}}=\frac{Q_{i, \mathrm{~L}}}{t_{\mathrm{flt}}}
$$

where $Q_{i, \mathrm{~L}}$ is the failure probability of LOTC from the $i$ th dispatchable configuration.

Thus, by substituting (3), (4), and (5) into (2), an expression for the TWA LOTC rate of the system may be obtained.

In a revision to the original SAE ARP5107 document, a revised method of calculating the time fractions $t_{i}, i=2, \ldots, n$ is given [4]. This method is claimed to balance better the fractions of time spent in each dispatchable system configuration because, rather than assuming the system is in the full-up state for all the time, it is assumed to be in the fullup state for $t_{1}$. The new values for $t_{i}$ are

$$
t_{i}=t_{1}\left(\lambda_{i} T_{i}\right)
$$

In this case (3) and (6) give a system of $n$ linear simultaneous equations that can be solved for $t_{i}$, $i=1, \ldots, n$. Then, together with (5) these may be substituted to give an expression for the TWA LOTC rate of the system.

\subsection{Reduced-state Markov model approach}

The reduced fault state Markov approach is similar to a conventional Markov modelling approach. However, there are two notable differences.

1. The number of system states is greatly reduced.

2. An artificial simulated repair transition is added to the model.

The reduced-state Markov approaches described in references $[\mathbf{1}],[3]$, and [4] are similar in that the number of system states is reduced by considering usually only single fault states, i.e. states where only one fault exists in the system in addition to the full-up state and the LOTC state. Dual and higher-order fault states may be added to the model if considered of particular importance or if the FADEC system architecture requires it. A reducedstate Markov model for a general system is shown in Fig. 5. This particular model is similar to that given in SAE ARP5107 revision 1 [4]. As a single mechanical/hydromechanical fault could cause LOTC from any fault state, a transition is added from the full-up (state 1) and all single fault states (states $2, \ldots, n$ ) to the LOTC state. The other addition to the model, over a conventional model, is the feedback loop, a simulated repair from the LOTC state to the full-up state. This is added to the model in order to allow a steady state solution to be calculated. Considering the Markov model shown in Fig. 5, the transition rate matrix, $\mathbf{A}$, is given by 


$$
\mathbf{A}=\left[\begin{array}{ccccc}
-\left(\lambda_{\mathrm{H}}+\sum_{i=2}^{n} \lambda_{i}\right) & \lambda_{2} & \cdots & \lambda_{n} & \lambda_{\mathrm{H}} \\
\nu_{2} & -\left(\nu_{2}+\lambda_{2, \mathrm{~L}}+\lambda_{\mathrm{H}}\right) & 0 & 0 & \lambda_{2, \mathrm{~L}}+\lambda_{\mathrm{H}} \\
\vdots & 0 & \ddots & 0 & \vdots \\
\nu_{n} & 0 & 0 & -\left(\nu_{n}+\lambda_{n, \mathrm{~L}}+\lambda_{\mathrm{H}}\right) & \lambda_{n, \mathrm{~L}}+\lambda_{\mathrm{H}} \\
\nu_{\mathrm{FB}} & 0 & \cdots & 0 & -\nu_{\mathrm{FB}}
\end{array}\right]
$$

All terms that are off-diagonal and are not elements of the first or last columns are zero. The term $\lambda_{\mathrm{H}}$ represents the sum of $\lambda_{\mathrm{HMC}}$ and $\lambda_{\mathrm{UR}}$. This leads to a system of $n+1$ differential equations

$$
\dot{\boldsymbol{Q}}(t)=\boldsymbol{Q}(t) \mathrm{A}
$$

where

$$
\boldsymbol{Q}(t)=\left[Q_{1}(t), Q_{2}(t), \ldots, Q_{n+1}(t)\right]
$$

and $Q_{i}(t)$ is the probability of the system being in state $i$ at time $t$. At steady state the rate of change of each of these probabilities is zero, therefore equation (8) becomes

$$
\mathrm{QA}=\boldsymbol{0}
$$

which is a system of $n+1$ linear simultaneous equations. These equations are dependent and, in order to obtain an independent system, one of the equations is arbitrarily chosen to be replaced by the constraint equation

$$
\sum_{i=1}^{n+1} Q_{i}=1
$$

In order to find the LOTC rate of the system, the average failure rate into the LOTC state, $n+1$, is

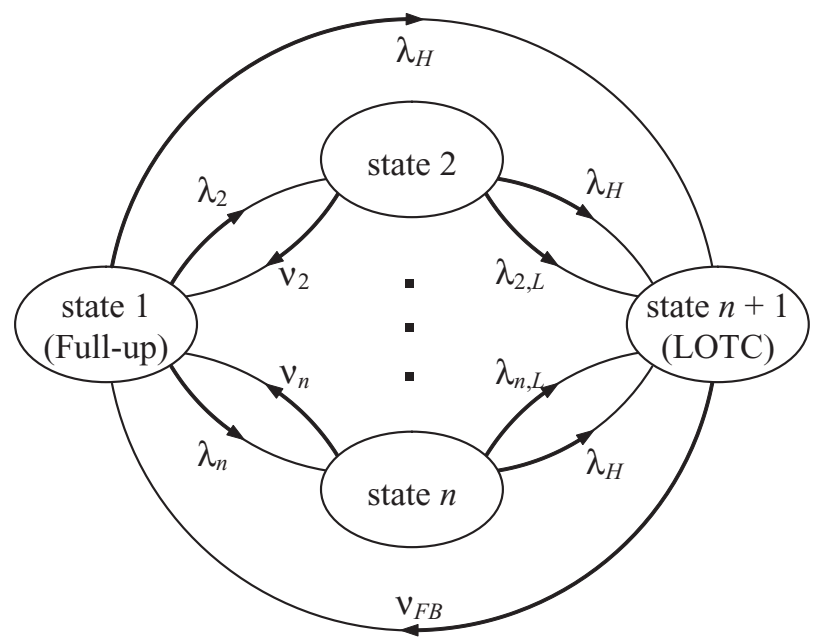

Fig. 5 A single fault state Markov model used. The definition of the reduced-state Markov (RSM) LOTC rate is hence

$$
\lambda_{\mathrm{RSM}}=\frac{\text { Probability flow into state } n+1}{1-\text { Probability of being in state } n+1}
$$

which is

$$
\lambda_{\mathrm{RSM}}=\frac{\lambda_{\mathrm{H}} Q_{1}+\sum_{i=2}^{n}\left(\lambda_{i, \mathrm{~L}}+\lambda_{\mathrm{H}}\right) Q_{i}}{1-Q_{n+1}}
$$

Equations 2 to $n$ of the set of simultaneous equations obtained above (from columns 2 to $n$ of matrix A) yield the following expressions for $Q_{i}$

$$
Q_{i}=\frac{\lambda_{i}}{\nu_{i}+\lambda_{i \mathrm{~L}}+\lambda_{\mathrm{H}}} Q_{1}, \quad i=2, \ldots, n
$$

which may be substituted into (13), along with a rearrangement of (11), to give

$$
\lambda_{\mathrm{RSM}}=\frac{\lambda_{\mathrm{H}}+\sum_{i=2}^{n}\left[\left(\lambda_{i} \lambda_{i, \mathrm{LH}}\right) /\left(\nu_{i}+\lambda_{i, \mathrm{LH}}\right)\right]}{1+\sum_{i=2}^{n}\left[\lambda_{i} /\left(\nu_{i}+\lambda_{i, \mathrm{LH}}\right)\right]}
$$

where $\lambda_{i, \mathrm{LH}}$ is the sum of $\lambda_{i, \mathrm{~L}}$ and $\lambda_{\mathrm{H}}$. This is the general form of the solution for the RSM model as given in reference [4]. If the repair intervals are as defined in equation (1), then the repair intervals are given as the reciprocals of the dispatch intervals, i.e.

$$
\nu_{i}=\frac{1}{T_{i}}
$$

Equations (15) and (16) may now be used to obtain the LOTC rate using different values of STD and LTD intervals.

\subsection{Monte Carlo simulation}

The first step in performing an MCS is to create a computer code that will model the behaviour of the system over time. The code contains a structured, logical set of rules that will describe how the system reacts to every event that may occur during its use [5]. When modelling TLD, such events could be component repairs, failures, sequences of failures, or TLD maintenance deadlines and the like. The generation of a uniform set of random numbers is key to the success of any MCS. In a TLD simulation these random numbers are used to generate component failure times using the relevant failure or repair distributions for each component. The simulations are run until such a time that the system fails or the 
maximum lifetime of the system is reached. After each simulation the relevant parameters are stored and, once these parameters lie within the required tolerance, the series of simulations is ended.

When TLD is modelled using MCS, the scheduling of events that affect the system is of the utmost importance. Component failure times are initially added to the schedule. The simulation time is advanced to the time of the first event chronologically in the schedule. As a component fails, the status of the system is checked and if the system fails, the simulation ends. If the system does not fail upon the failure of a component, then a list of TLD criteria is checked to see if a TLD maintenance deadline or periodic inspection should be added to the schedule because of the component failure. The deadline or inspection could be added because of that failure alone, or because of that failure acting in combination with other component failures. The schedule and the correct ordering of events is perhaps the most important part of the simulation of TLD. The structure utilized in the MCS code used here stores the positions of events previous to and after each event and these must be updated as, for example, the first event chronologically is removed from the schedule, or deadlines are removed from the schedule after faults are repaired.

One of the major advantages of implementing an MCS is the large degree of flexibility or complexity that may be involved in the code. For instance, when a maintenance deadline is reached, the different repair strategies that are possible may be carried out easily. It would be possible, for example, to clear all faults from the system at each deadline, to clear all faults falling into the same dispatch category, or simply to address the fault that caused the deadline. Other strategies are possible and part of the beauty of MCS lies with the fact that different maintenance strategies could be tested before being applied to a real system. MCS is also able to model different strategies better than the usual TWA or Markov-based approaches. Different flight lengths to represent short-haul and long-haul operations can be specified, as can different system operational lifetimes.

The algorithm for the main module of the MCS code used in this work is shown in Fig. 6. Owing to the fact that any maintenance operations (TLD deadlines and inspections) cannot occur mid-flight, the time of such operations are adjusted to occur between flights. This is done by moving the operation forward to the beginning of the flight in which it would otherwise fall.

The code written and used to model TLD here is flexible in that it can use data from any fault tree of a system failure mode. In addition to the fault tree, the failure distributions of the components and the TLD dispatch criteria to be applied to the system are passed to the code before simulations begin. The failure rate of the system is calculated after every 1000 simulations and the solution is obtained to the required number of significant figures. This is done by checking that the value of the failure rate is unchanged for a number of consecutive calculations.

\section{EXAMPLE SYSTEM}

The system modelled in this work is a simple one, containing only four components. The system architecture is shown in Fig. 7. As can be seen from the diagram, it essentially consists of two channels, 1 and 2, each of which contains a power supply and a CPU. However, in order to add further redundancy to the system, a link between the two channels is provided that allows, for example, the channel 1 power supply to provide power to the channel 2 CPU if its own power supply fails. The failure rates of the individual components are given in Table 1, along with the dispatch category that will be applied if one of them fails. These dispatch categories were determined for each component by considering the instantaneous failure rate (to LOTC) with that component failed, using the approximation given in equation (5). The same approximation was used to estimate the instantaneous failure rate (to LOTC) of each of the dual fault states, i.e. the system configurations where two faults are present in the system. These failure rates suggested that all dual faults would fall into the DND category and thus need not be included in the SAE TWA and Markov analyses. However, these dual faults are included in the MCS as DND faults. Higher-order fault states than this need not be considered for this system as, once three components are failed, the system is definitely failed.

When modelling the system using the TWA and RSM approaches, the failure rate due to mechani$\mathrm{cal} /$ hydromechanical faults, $\lambda_{\mathrm{HMC}}$, and the failure rate due to unrevealed faults, $\lambda_{\mathrm{UR}}$, were not considered in the SAE analyses.

TWA results were obtained for both methods of calculating the time fractions; see equations (4) and (6), given in the original [3] and revised [4] versions of SAE ARP5107. As mentioned previously, the Markov results were obtained for a single fault state model, as higher-order faults would fall into the DND category and need not be included in the analysis. This is also the case for the TWA model but these DND category faults are included in the MCS model. Results were obtained for the MCS for a number of different maintenance strategies and approaches. STD and LTD faults were addressed using all possible combinations of the MEL and PIR maintenance approaches. Thus, both STD and LTD 


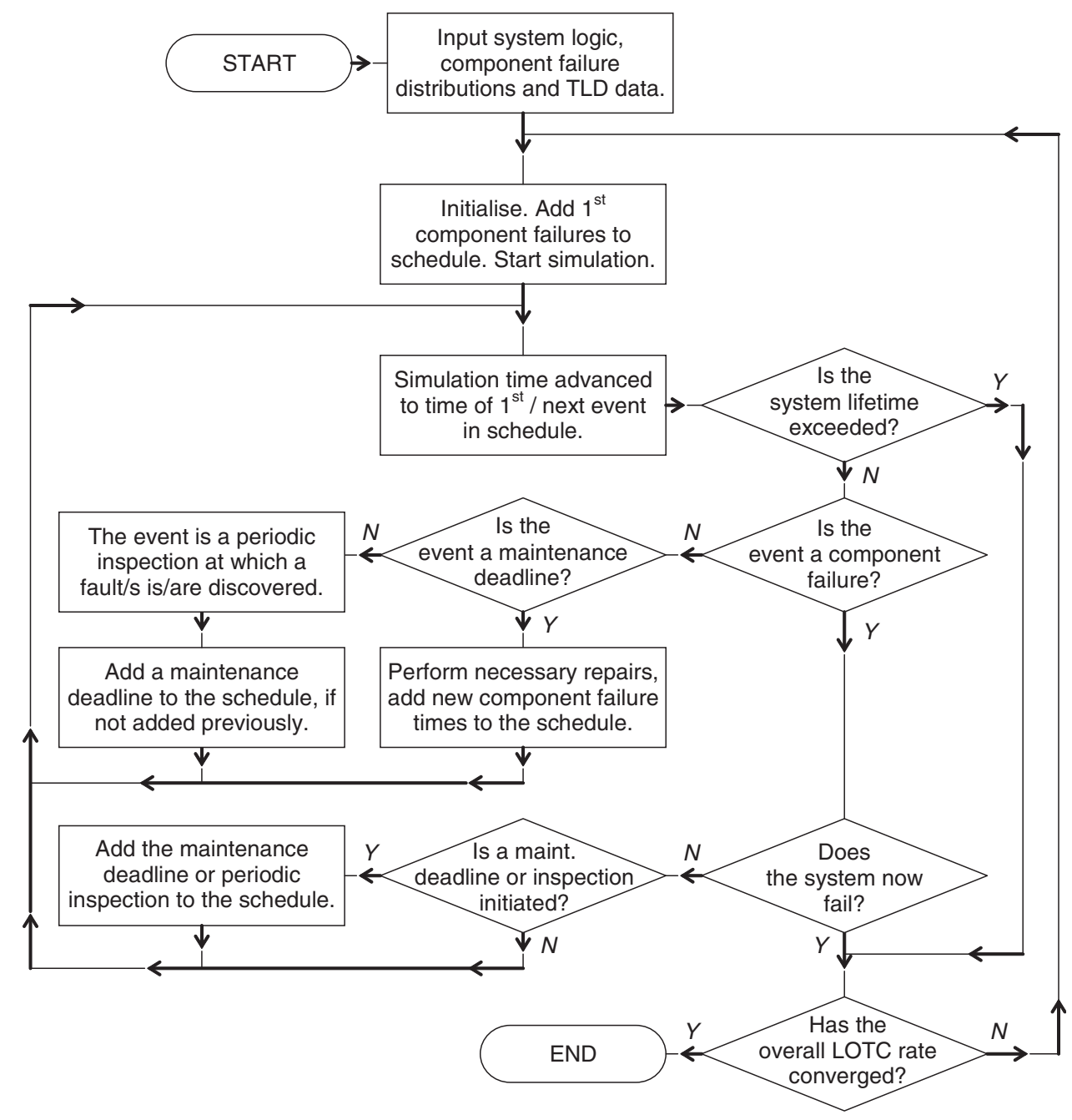

Fig. 6 The algorithm for the main module of the MCS code

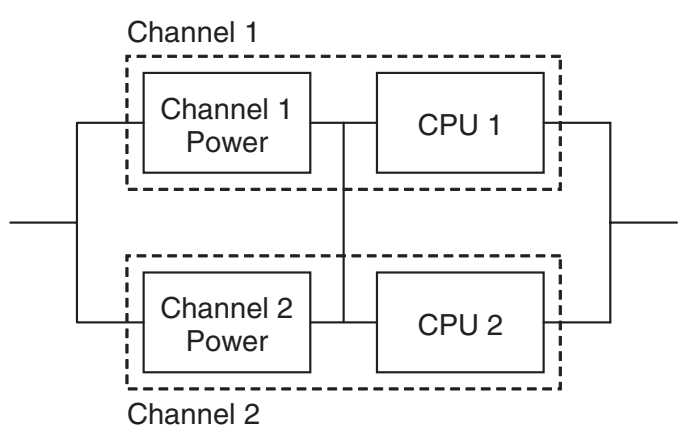

Fig. 7 The simple FADEC system representation

faults were dealt with using MEL maintenance in one set of simulations, one with MEL and the other with PIR in the next set, and, finally, both would be maintained using PIR maintenance. When PIR maintenance was used to maintain faults, the inspection interval was varied as a function of the dispatch interval and defined as 1.0, 1.5, and, finally, 2.0 times the dispatch interval. At the maintenance
Table 1 Component failure rates and dispatch categories

\begin{tabular}{lll}
\hline Component & Failure rate $(\mathrm{f} / \mathrm{h})$ & Dispatch category \\
\hline Power 1 & $9.0 \times 10^{-5}$ & STD \\
CPU 1 & $5.2 \times 10^{-5}$ & LTD \\
Power 2 & $8.0 \times 10^{-5}$ & STD \\
CPU 2 & $6.5 \times 10^{-5}$ & LTD \\
\hline
\end{tabular}

deadlines, the faults could be repaired in a number of different ways in order to allow further dispatch after the deadline. For this system the repairs were carried out in three different ways, each of which represented carrying out a varying amount of work on the system.

1. Repair the last fault of the group of faults that initiated the maintenance deadline.

2. Repair all of the group of faults that initiated the maintenance deadline.

3. Clear all faults present in the system at maintenance deadlines. 
The first of these maintenance approaches represents perhaps the minimum amount of work that could be carried out at a maintenance deadline in order to allow further dispatch. An example of applying this approach would be as follows. If CPU1 failed, an LTD interval would be initiated. If Powerl was to fail in the subsequent period before the maintenance deadline, a DND maintenance deadline would be added to the end of the current flight. In this maintenance approach, Powerl would be repaired, leaving the CPU fault present in the system until the LTD maintenance deadline occurs. The second approach would be a slightly more rigorous approach to repairs than the first because, for instance, in the example given above, as the DND maintenance deadline was encountered, all faults that caused it, i.e. Powerl and CPU1, would be repaired. The final maintenance approach listed above is most likely to be the one that will produce the lowest LOTC rate, as when any maintenance deadline occurs, all faults that are present in the system are repaired and the system is thus returned to a full-up state. For the MCS the maximum lifetime of the system was assumed to be $200000 \mathrm{flt} \mathrm{h}$. This corresponds to a period of use of approximately 37 years for a system used for $15 \mathrm{~h} /$ day. The length of a flight (used in the TWA approach and the MCS) was set to be $5 \mathrm{~h}$. This was assumed in a worked example in reference $[\mathbf{1}]$.

\section{RESULTS}

Results were obtained for an STD interval length of between 50 and $200 \mathrm{flt} \mathrm{h}$ in $50 \mathrm{flt} \mathrm{h}$ steps with the length of the LTD interval varying between 200 and $2000 \mathrm{flt} \mathrm{h}$ in $200 \mathrm{~h}$ increments. Below are some of the results obtained for the $200 \mathrm{flt}$ h STD interval.

Figure 8 shows a comparison of the results from the SAE approaches and MCS with STD faults addressed using the MEL maintenance approach, and LTD faults addressed using the PIR maintenance approach, which appears to be the most commonlyused maintenance combination in practice. The PIR inspection intervals are set at 1.5 times the dispatch intervals and at maintenance deadlines, all faults are cleared from the system (the third of the approaches listed earlier). It can be seen from this graph that the TWA method with the original time fraction coefficients produces the highest calculated LOTC rate and that the TWA method with the revised time fraction coefficients produces a calculated LOTC rate that is consistently lower than this over the range of LTD intervals. The single state Markov is again lower but the lowest calculated LOTC rate comes from the MCS. Assuming that the MCS models the system more accurately than the other models, which is a reasonable assumption, this means that the TWA and single fault state Markov methods of analysis are suitable models for this system because the LOTC rate is overestimated.

Figure 9 shows a comparison of results for the three different maintenance approaches described earlier. Again, STD faults are addressed using MEL maintenance and LTD faults are addressed using PIR maintenance with the inspection interval set at 1.5 times the dispatch interval. This graph shows that there is a significant difference in the predicted LOTC rate for the three maintenance approaches. While repairing the last fault of the group that

(STD-MEL, LTD-PIR) inspection interval = 1.5x dispatch interval. STD interval $=200 \mathrm{hrs}$.

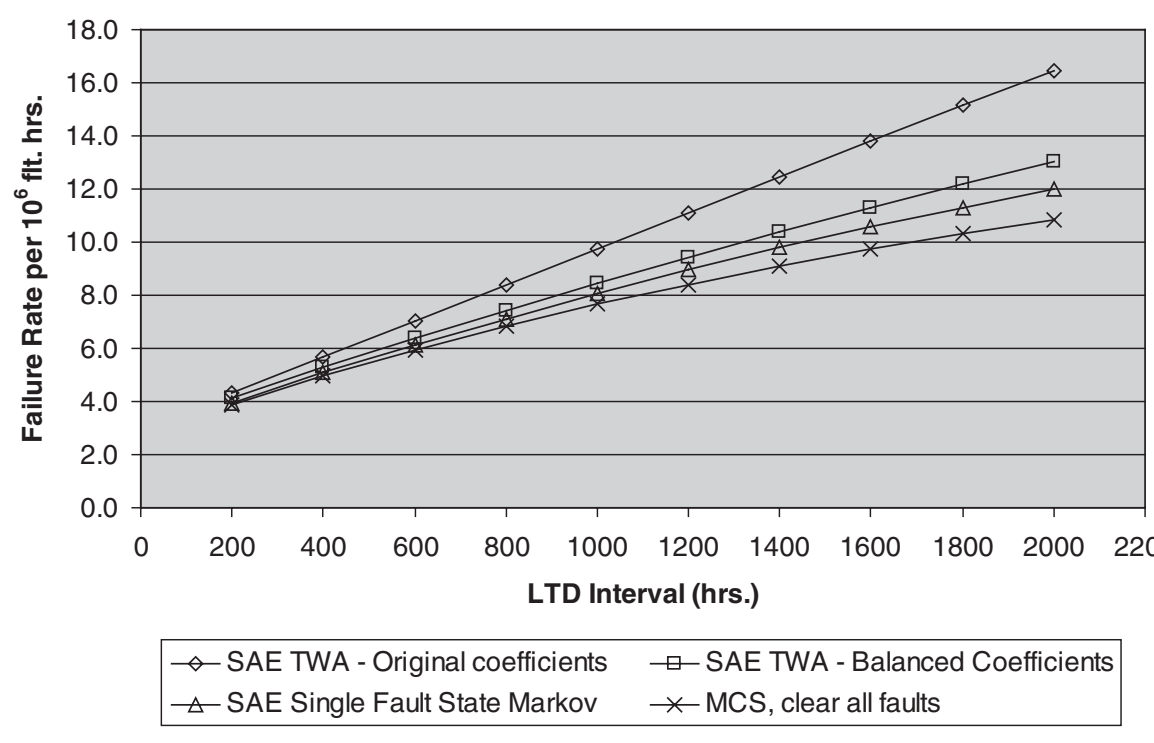

Fig. 8 A comparison of the SAE ARP5107 approaches with MCS 
(STD-MEL, LTD-PIR) inspection interval = 1.5x dispatch interval. STD interval $=200 \mathrm{hrs}$.

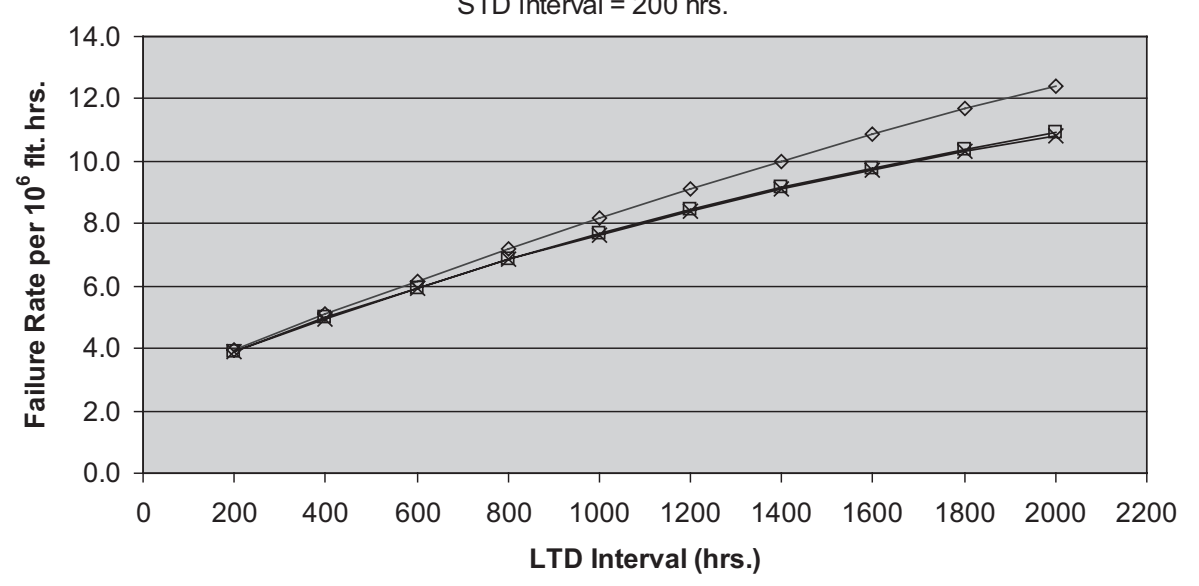

$\smile$ Repair the last fault of the group of faults that initiated the deadline
$\square-$ Repair all of the group of faults that initiated the deadline
$\star \leftarrow$ Clear all faults present in the system at maintenance deadlines

Fig. 9 A comparison of MCS results for different maintenance approaches

caused the deadline, the predicted LOTC rate is greater than the LOTC rate when repairing all faults that initiated the deadline or clearing all faults present in the system at the maintenance deadlines. The final two maintenance approaches give very similar results, the LOTC rate obtained when all faults are cleared from the system at maintenance deadlines being slightly lower. One would expect that this particular difference could be even more pronounced if a larger system were being modelled. With this in mind consider Fig. 10, which shows a comparison of the MCS results for the best and worst of the maintenance approaches with the results from the TWA method (with balanced time coefficients) and the single fault state Markov method. It can be seen here that the single fault state Markov approach actually gives an LOTC rate that is lower than that predicted when the system is repaired by repairing only the last fault of the group causing a maintenance deadline. The TWA method still overestimates the LOTC rate, even for this maintenance approach. As observed earlier, both SAE methods overestimate the LOTC rate when all faults are cleared from the system at maintenance deadlines. In order better to quantify these differences, consider Figs 11 and 12, which show the percentage differences of the LOTC rate obtained using the TWA and single fault state Markov methods from the predicted LOTC rate from the MCS. Figure 11 clearly shows that when maintenance involves clearing all faults from the system, the TWA method (with the original time coefficients) overestimates the LOTC rate in comparison to the MCS by between 11.7 per cent and 50.4 per cent over the LTD interval range of 200 to $2000 \mathrm{flt} \mathrm{h}$. Over the same range the TWA method with the balanced time coefficients overestimates the LOTC rate by between 5.7 per cent and 18.8 per cent and the single fault state Markov model overestimates the LOTC rate by between 1.75 per cent and 9.68 per cent. Figure 12 shows that the TWA method with original time coefficients overestimates the LOTC rate in comparison to the MCS by between 7.2 per cent and 27.6 per cent over the LTD interval range between 200 and $2000 \mathrm{flt} \mathrm{h}$. The TWA method with balanced time coefficients overestimates very slightly, the percentage overestimate actually falling from 1.5 per cent to 0.8 per cent. However, the single fault state Markov approach underestimates the LOTC rate in relation to the MCS by 2.3 per cent at a value of $200 \mathrm{flt} h$ for the LTD interval. This percentage rises to 6.9 per cent at a value of $2000 \mathrm{flt} h$ for the LTD interval.

The question now is, what effect these differing modelling approaches would have on the dispatch of the system considered as an example here. Considering again Fig. 8, it can be seen that, given the upper limit for the average LOTC rate of ten failures per $10^{6} \mathrm{flt} \mathrm{h}$ that, given a STD interval of $200 \mathrm{~h}$, the LTD interval could be set at about $1050 \mathrm{~h}$ if the TWA method (with the original time coefficients) was used to model the system. Using the same method, but with the balanced coefficients, would allow the LTD interval to be set at about $1300 \mathrm{~h}$, a vast improvement. However, the single fault state Markov model would allow the LTD interval to be set at about $1450 \mathrm{~h}$ and the MCS would allow a dispatch interval for LTD faults of around $1650 \mathrm{~h}$ if all faults were to be cleared from the system at the 
(STD-MEL LTD-PIR) inspection interval $=1.5 \mathrm{x}$ dispatch interval. STD interval $=200 \mathrm{hrs}$.

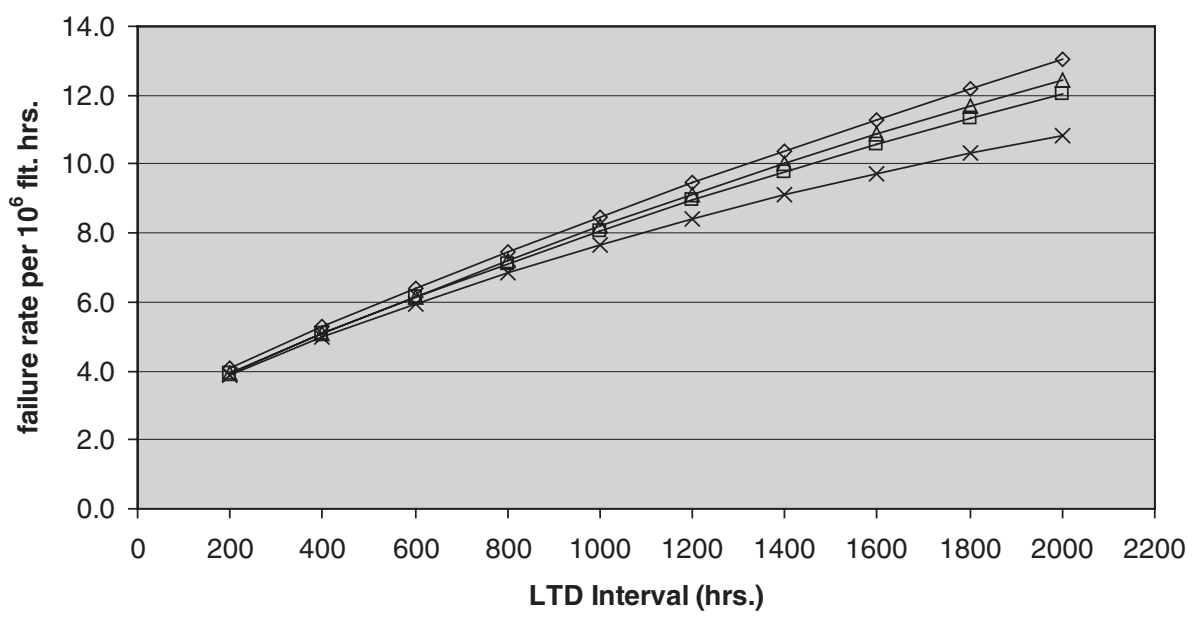

\begin{tabular}{ll|}
\hline$\diamond$ SAE TWA - Balanced Coefficients & $\square$ SAE Single Fault State Markov \\
$\triangleleft$ MCS, repair last fault initiating deadline & $\star$ MCS, clear all faults \\
\hline
\end{tabular}

Fig. 10 A comparison of differing maintenance in MCS with SAE ARP5107 approaches

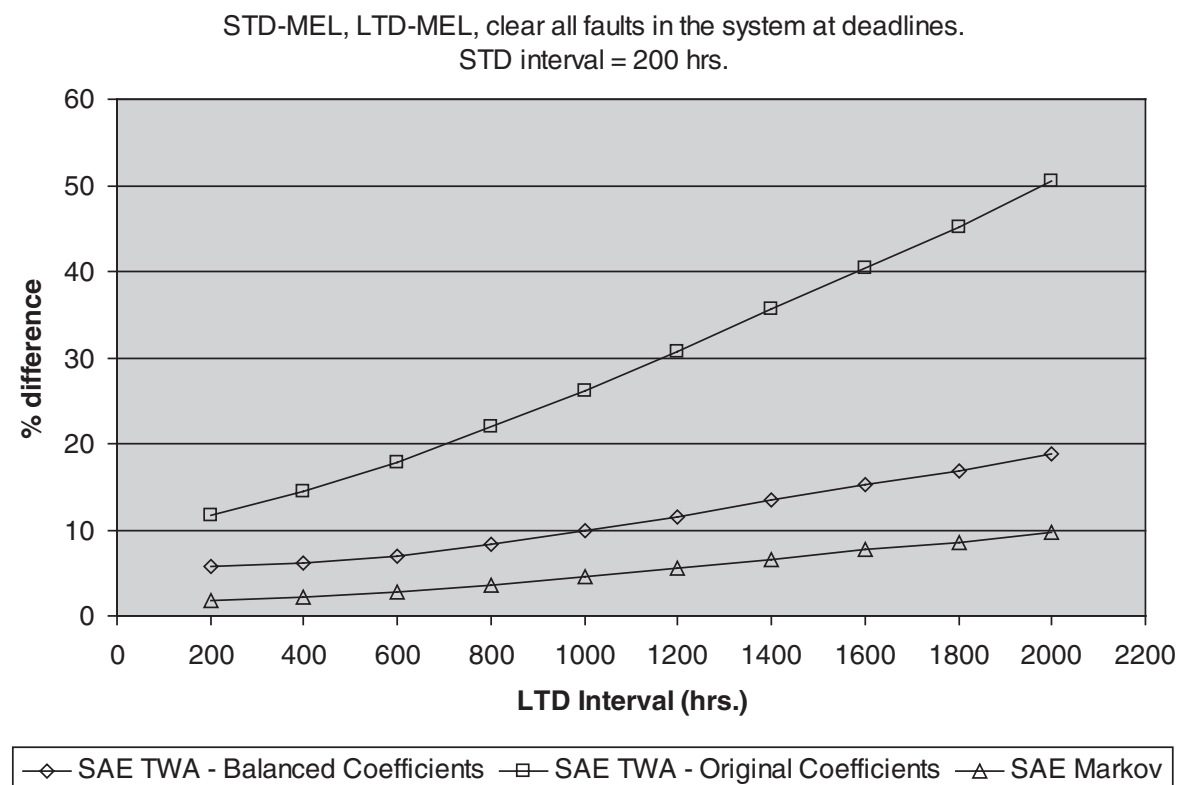

Fig. 11 Percentage difference of SAE approaches from MCS

same time. However, from Fig. 9 it can be seen that, if the maintenance approach involves repairing the last fault of the group that initiated the deadline, the maximum allowed LTD dispatch interval would be around $1400 \mathrm{~h}$.

Only a small sample of the MCS results obtained are given because all the results showed similar trends to those presented above and the scenarios involved here were considered to be a good representation of a general approach to TLD. The shorter the STD interval, the smaller was the LOTC rate for particular LTD interval values, as would be expected. As would also be expected, increasing the length of the inspection interval when PIR maintenance was used led to an increase in the LOTC rate of the system. This increase in the LOTC rate was most pronounced when PIR was used to address LTD category faults, regardless of the maintenance approach used for STD faults. This may be due to the difference in length of the dispatch intervals 
STD-PIR, LTD-PIR, repair last fault of the group that initiated the deadline. STD interval $=200 \mathrm{hrs}$.

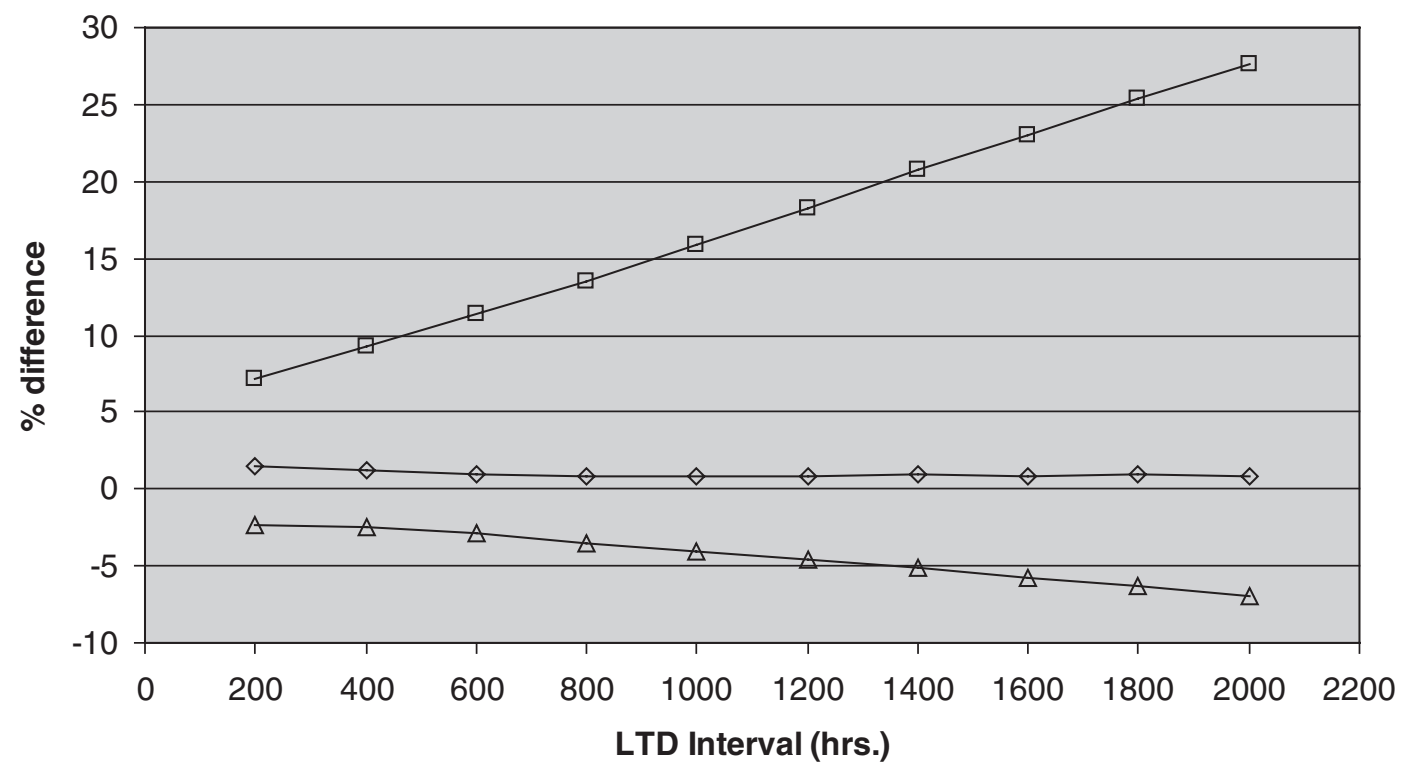

$\diamond$ SAE TWA - Balanced Coefficients $\square-$ SAE TWA - Original Coefficients $\triangle$ SAE Markov

Fig. 12 Percentage difference of SAE approaches from MCS

themselves and therefore the relative lengths of the inspection intervals. For instance, for the maximum modelled LTD interval of $2000 \mathrm{~h}$, the inspection interval (and hence potential exposure time of this failed system state to further faults) would be 4000 h. Contrast this with the situation for the maximum modelled STD interval of $200 \mathrm{~h}$, where the inspection interval would only be $400 \mathrm{~h}$.

\section{CONCLUSIONS}

A number of conclusions may be drawn from the modelling of the use of TLD on this simple system. The first, and perhaps most important, is that MCS allows the flexibility to model a large number of potential maintenance scenarios and observe the effect of these on the LOTC rate of the system. In short, a more exact modelling of the system lifetime is obtained. One could expect MCS to provide a more accurate measure of the LOTC rate than that obtained using the SAE approaches in general, for a number of reasons. First, the construction of the TWA and RSM models involve considering only a limited number of dispatchable system states, nondispatchable states not being included in the models. This could result in a model that does not describe the system in a completely accurate manner. Contrast this with MCS, where a complete system fault tree is used and no eventualities need be discounted when calculating system LOTC rates. Failure rates must be calculated for use in each of the SAE models, both into and out of the dispatchable system states. An issue here is that these failure rates could be difficult to calculate, particularly if one considers the fact that there may be dependencies between various aircraft subsystems. Another point to take into account when one considers failure rates is that those of individual components may vary with time. A property such as this can be incorporated easily into an MCS approach, whereas it could prove difficult to include in other approaches.

For this system the different maintenance approaches were demonstrated to have such an effect on the LOTC rate that the single fault state Markov approach, for one such maintenance approach, was seen to underestimate the LOTC rate of the system in comparison to the MCS. It should be noted that the maintenance approach in question (repairing the last fault of the group of faults that caused the deadline) may not necessarily be a realistic approach and that it may be possible to modify the single fault state Markov approach in order better to model such a repair strategy. However, this may bring an element of doubt as to whether the SAE modelling approaches would always guarantee a LOTC rate of 10 or less failures per $10^{6} \mathrm{~h}$ for any system. Of course, the application of TLD to a more complex, realistic system may result in more accurate results or, at least, a guaranteed overestimate 
of the LOTC rate of the system. This requires further investigation.

Clearly, the use of MCS could prove to be a useful tool in the modelling of TLD. Indeed, if one looks from the certification viewpoint, MCS could allow the demonstration of compliance of the LOTC rate, while being able to maximize/optimize the dispatch intervals in order to establish the most advantageous maintenance strategy. MCS could also offer the most accurate measure for the LOTC rate of the system and be used to obtain other information about the system, such as the instantaneous LOTC rates from various system states.

\section{ACKNOWLEDGEMENT}

The authors would like to thank Phil Wilkinson, Rolls-Royce Derby, for the advice given during the completion of the work described in this paper.

\section{REFERENCES}

1 Larsen, H. and Horan, G. Time-limited dispatch: an interactive training and self-study course. Keybridge Technologies, Inc., 2002.

2 FAA Memorandum: policy for time-limited dispatch (TLD) of engines fitted with full authority digital engine control (FADEC) systems. Policy No. ANE1993-33.28TLD-R1, 29 June 2001.

3 SAE ARP 5107, Guidelines for time-limited-dispatch (TLD) analysis for electronic engine control systems, 1997 (SAE International).

4 SAE ARP 5107, Guidelines for time-limited-dispatch (TLD) analysis for electronic engine control systems, Revision 1, scheduled release: December 2004 (SAE International).

5 Andrews, J. D. and Moss, T. R. Reliability and risk assessment (2nd edn), 2002 (Professional Engineering Publishing, Bury St Edmunds and London).

\section{APPENDIX}

\section{Notation}

O null vector

A transition rate matrix

DND do not dispatch

FADEC full authority digital electronic control

LOTC loss of thrust control rate

LTD long time dispatch

MCS Monte Carlo simulation

MEL minimum equipment list

PIR periodic inspection and repair

$\boldsymbol{Q}(t) \quad$ vector of state probabilities at time $t$

$Q_{i}(t) \quad$ probability of state $i$ at time $t$

$Q_{i, \mathrm{~L}} \quad$ failure probability of LOTC from $i$ th dispatchable system configuration

STD short time dispatch

$t_{\mathrm{flt}} \quad$ flight time (hours)

$t_{i} \quad$ fraction of time dispatching from dispatchable system configuration $i$

$T_{i} \quad$ dispatch interval for the $i$ th dispatchable system configuration (hours)

$T_{\text {LTD }} \quad$ long time dispatch interval (hours)

$T_{\text {STD }} \quad$ short time dispatch interval (hours)

$\lambda_{\mathrm{H}} \quad$ sum of $\lambda_{\mathrm{HMC}}$ and $\lambda_{\mathrm{UR}}$

$\lambda_{\mathrm{HMC}}$ sum of failure rates due to mechanical/ hydromechanical faults

$\lambda_{i} \quad$ failure rate into the $i$ th dispatchable system configuration

$\lambda_{i, \mathrm{~L}} \quad$ system failure rate to LOTC from the $i$ th dispatchable system configuration

$\lambda_{i, \mathrm{LH}} \quad$ sum of $\lambda_{\mathrm{H}}$ and $\lambda_{i, \mathrm{~L}}$

$\lambda_{\text {RSM }} \quad$ reduced-state Markov model LOTC rate

$\lambda_{\text {TWA }}$ time-weighted average model LOTC rate

$\lambda_{\mathrm{UR}} \quad$ sum of failure rates due to unrevealed electrical/electronic faults

$\nu_{\mathrm{FB}} \quad$ feedback repair rate

$\nu_{i} \quad$ repair rate from the $i$ th dispatchable system configuration 